\title{
Measurement Campaign of Rural Overhead MV Power Distribution Networks for PLC Systems
}

\author{
Thiago F. do A. Nogueira, Thiago R. Oliveira, Antônio A. M. Picorone, Mateus de L. Filomeno, \\ Ândrei Camponogara, Antônio C. S. Lima, and Moisés V. Ribeiro
}

\begin{abstract}
This paper investigates the suitability of the inductive coupling-based narrowband power line communication systems in overhead medium-voltage power distribution networks. Relying on raw data obtained from a measurement campaign, which was carried out in rural circuits in the South of Brazil, it is shown that power line channel impulse responses associated with these distribution networks can be modeled as time-invariant systems while the additive noise can be modeled as a white Gaussian random process. Also, reasonable achievable data rates are noted.
\end{abstract}

Keywords-measurement campaign, medium-voltage, power distribution network, power line communication.

\section{INTRODUCTION}

The feasibility and usefulness of narrowband power line communication (PLC) systems over overhead medium-voltage power distribution networks (MV-PDNs) have been investigated as an opportunity of furthering communications media for electric utilities, seizing the already existing deployed network. The use of MV-PDN for data communication purposes has been investigated since the beginning of the last century [1]. However, one century later, renewed interests in narrowband PLC systems over overhead MV-PDNs emerged once these networks can be regarded as a pervasive telecommunication infrastructures, this way meeting the needs and demands of electric utilities (e.g., smart grid) [2].

Several researches have pointed out that the deployment and operational costs of PLC systems can be subsidized because electric power systems are already installed everywhere, which is argued as a competitive advantage in favor of the deployment of PLC systems [3], [4]. In this sense, PLC

This study was financed in part by the Coordenação de Aperfeiçoamento de Pessoal de Nível Superior - Brasil (CAPES) - Finance Code 001, Copel Distribuição LTD - PD2866-0420/2015, CNPq, Federal University of Juiz de Fora, FAPEMIG, and INERGE.

Thiago F. do A. Nogueira, Mateus de L. Filomeno, Ândrei Camponogara, and Moisés V. Ribeiro are with the Department of Electrical Engineering, Federal University of Juiz de Fora, Juiz de Fora 36036 330, Brazil. e-mails: \{thiago.nogueira, mateus.lima, acamponogara, mribeiro\} @engenharia.ufjf.br) Thiago R. Oliveira is with the Electronics Department, Federal Institute of Education, Science and Technology of the Southeast of Minas Gerais (IFSEMG), Campus Juiz de Fora, Brazil. e-mail: thiago.oliveira@ifsudestemg.edu.br

Antônio A. M. Picorone is with Production Engineering and Mechanics Department, Federal University of Juiz de Fora, Juiz de Fora, MG 36036 330, Brazil. e-mail: antonio.picorone@engenharia.ufjf.br

Antonio C. S. Lima is with the Department of Electrical Engineering, Federal University of Rio de Janeiro, Rio de Janeiro 21941 901, Brazil. e-mail: acsl@poli.ufrj.br systems have been applied for supporting Smart Grid (SG), the Internet of Things (IoT), and Industry 4.0 applications [5].

In the literature, there is a clear lack of insights about the advantages and disadvantages related to the use of inductive coupling-based narrowband PLC systems for overhead MV-PDNs. Actually, the usage of capacitive couplers is more common than inductive ones, mainly due to the known limitations of the latter in comparison to the former as well reported in [6]. For instance, to the best of authors' knowledge, there is no statistical analyses based on measurement campaigns, which are required to characterize the behavior of MV-PDN and the impact of the additive noise in real conditions.

In this regard, this work investigates the adequacy of overhead MV-PDNs as a data communication media for narrowband PLC systems that make use of inductive coupling devices. In this sense, it relies on a measurement campaign that was carried out in overhead MV-PDNs, located in Brazilian rural areas. The chosen frequency bandwidth, which is between $100 \mathrm{kHz}$ and $500 \mathrm{kHz}$, complies with the IEEE standard for narrowband PLC systems [7]. Based on the data collected in the measurement campaign, we conclude that the PLC channel impulse responses associated with overhead MV-PDN can be modeled as time-invariant systems in rural areas while the additive noise can be modeled as a white Gaussian random process. Moreover, the numerical results show reasonable achievable data rates.

The rest of this paper is organized as follows: Section II presents the formulation adopted in the analysis of the problem. Section III describes the measurement setup and campaign. Section IV analyzes numerical results. Concluding remarks are presented in Section V.

\section{Problem Formulation}

An illustration of a PLC system operating over a MV-PDN is shown in Fig. 1. Based on this illustration, the inductive coupling devices constitute the interface between the PLC devices and medium-voltage (MV) power cables. These kinds of coupling devices are, at the first sight, very convenient because they can be installed over an energized MV-PDN. Also, Tx and Rx represent transmitter and receiver devices that make use of one PLC coupling device per phase.

A PLC system over MV-PDN is designed for data communications in the frequency band delimited by 0 and $B \mathrm{~Hz}$ (i.e., bandlimited and baseband data communications) and the MV-PDN can be modeled as a random system. The 
received signal is expressed as

$$
Y_{P}(t)=\tilde{Y}_{P}(t)+V_{P}(t)=\int_{-\infty}^{+\infty} h_{P}(t, \tau) X(\tau) d \tau+V_{P}(t)
$$

where $X(t) \in \mathbb{R}$ represents the transmitted signal, modeled as a wide sense stationary stochastic process; $h_{P}(t, \tau) \in \mathbb{R}$ is the time-varying channel impulse response (CIR), which models the propagation of the transmitted signal through the MV-PDN; $V_{P}(t) \in \mathbb{R}$ is the additive noise, modeled as a wide sense stationary random process; and $\tilde{Y}_{P}(t) \in \mathbb{R}$ and $Y_{P}(t) \in \mathbb{R}$ denote wide sense stationary random processes for the free-of-noise received signal and the received signal, respectively.

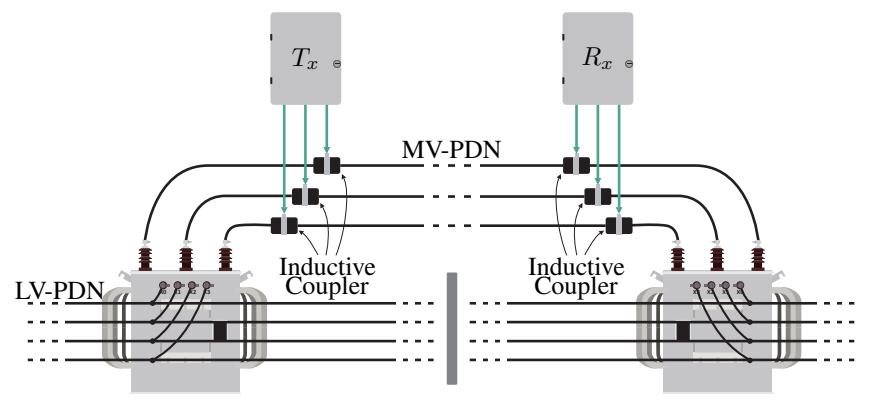

Fig. 1: An illustration of an inductive coupling-based PLC system over a MV-PDN.

The random linear periodically time-varying (LPTV) behavior of PLC channels means that $h_{P}(t, \tau)$ defines a cyclostationary process with a period equal to half of the mains frequency. In this sense, based on the knowledge of the coherente time ${ }^{1}$ of PLC channels, $T_{c} \in \mathbb{R}_{+}$, the mains cycle $(50 \mathrm{~Hz}$ or $60 \mathrm{~Hz})$ can be divided into microslots of time interval duration equal to $T_{m} \in \mathbb{R}_{+} \mid T_{m}<T_{c}$ and, as a consequence, the PLC channels can be modeled as linear time-invariant (LTI) during the time interval duration of one microslot [8]. In other words, the CIR of a PLC channel during the $i^{\text {th }}$ microslot can be expressed as

$$
h_{P, i}(t), \forall t \in\left[i T_{m},(i+1) T_{m}\right] \mid T_{m} \leq T_{c} .
$$

For the sake of simplicity, the index $i$ is dropped. Consequently, the discrete-time representation of the CIR of the PLC channel in a single microslot is given by

$$
h[n]=\left.h_{P}(t)\right|_{t=n T_{s}}, n=0,1, \ldots, L_{h}-1,
$$

where $L_{h}$ is the length of this LTI channel, $T_{s}=1 / f_{s}$ is the sampling period and $f_{s}=2 B \mathrm{~Hz}$ is the sampling frequency.

\section{MeAsurement CAMPAign}

The measurement campaign was carried out in $13.8 \mathrm{kV}$ MV-PDNs, located in a rural area, belonging to a Brazilian electric utility, which is called Companhia Paranaense de Energia (COPEL), in the city of Curitiba, Parana State, Brazil. The main characteristics of the rural feeder are listed in TABLE I. A measurement setup was specially designed for

\footnotetext{
${ }^{1}$ The coherence time denotes the maximum time interval in which the characteristics of the channels face irrelevant changes.
}

TABLE I: Main characteristics of the Rural Feeder.

\begin{tabular}{|c|c|}
\hline Characteristics & Rural Feeder \\
\hline \hline Service Characteristic & Urban and rural \\
\hline Substation Origin & Campo Comprido-230 kV \\
\hline Operation Voltage & $13.8 \mathrm{kV}$ \\
\hline Nominal Current & $135 \mathrm{~A}$ \\
\hline \multirow{2}{*}{ Network Morphology } & $1^{*}, 2^{*}$ \\
\cline { 2 - 2 } Covering Municipality & Curitiba and Campo Magro \\
\hline \multirow{3}{*}{ Consumers } & Residential: 2,596 \\
\cline { 2 - 2 } & Commercial: 243 \\
\cline { 2 - 2 } Electrical equipment & Industrial: 80 \\
\cline { 2 - 2 } & Transformers: 271 \\
\cline { 2 - 2 } & Switches: 117 \\
\cline { 2 - 2 } & Capacitors: zero \\
\hline \multirow{3}{*}{ Length } & $55,934.25$ meters \\
\hline \multirow{2}{*}{$1^{*}-$ Bare cables (conventional) } \\
\multirow{2}{*}{$2^{*}-$ Protected cables (compact network of distribution) }
\end{tabular}

carrying out the measurement campaign. The implemented solution makes use of inductive coupling devices for injecting and extracting PLC signals to and from the MV-PDNs, and is divided into two parts, the transmission and reception setups. The block diagrams of these setups are illustrated in Fig. 2.

The inductive coupling devices installed in the energized MV power cables are shown in Fig. 3. The components of both transmission and reception setups are grounded before the connection to the installed coupling devices. Regarding the transmission setup, the demultiplexer input connects to the power amplifier output, which has its input connected to the output of the signal generator. Also, the signal generator connects to the rugged computer through an ExpressCard interface. In the reception scenario, the multiplexer output is connected to a bandpass filter, which has its output connected to the input of the signal digitizer board. The signal digitizer is boarded into a rugged computer responsible for managing and monitoring the data acquirement.

In the course of the measurement campaign, five circuits were considered for performing the measurements. Also, phases $\mathrm{A}, \mathrm{B}$, and $\mathrm{C}$ were taken into account during the measurement campaign. The distances of the MV-PDN circuits varied from hundreds of meters to few kilometers. Moreover, a measure of PLC channels in a circuit produces 1,953 successive estimates of channel frequency responses (CFRs). In each circuit, three measures of PLC channels were performed per phase, resulting in a total of 5,859 CFR estimates per phase. Altogether, 76,167 CFR estimates were collected from the rural area. Regarding the additive noise, one measure with $3 \times 10^{6}$ samples per phase and circuit were obtained, resulting in 5 raw data from the rural area. Note that each additive noise raw data corresponds to a time interval of 3 seconds.

\section{NuMERICAL RESUlTS}

The numerical results presented in this section were obtained through the raw data provided by the measurement campaign, which was detailed in Section III. The frequency range from $B_{L}=100 \mathrm{kHz}$ up to $B_{H}=500 \mathrm{kHz}$ was adopted because the inductive coupling device operates in this frequency band. The data rate analysis is based on the average channel attenuation (ACA) values of CFRs and the estimates 


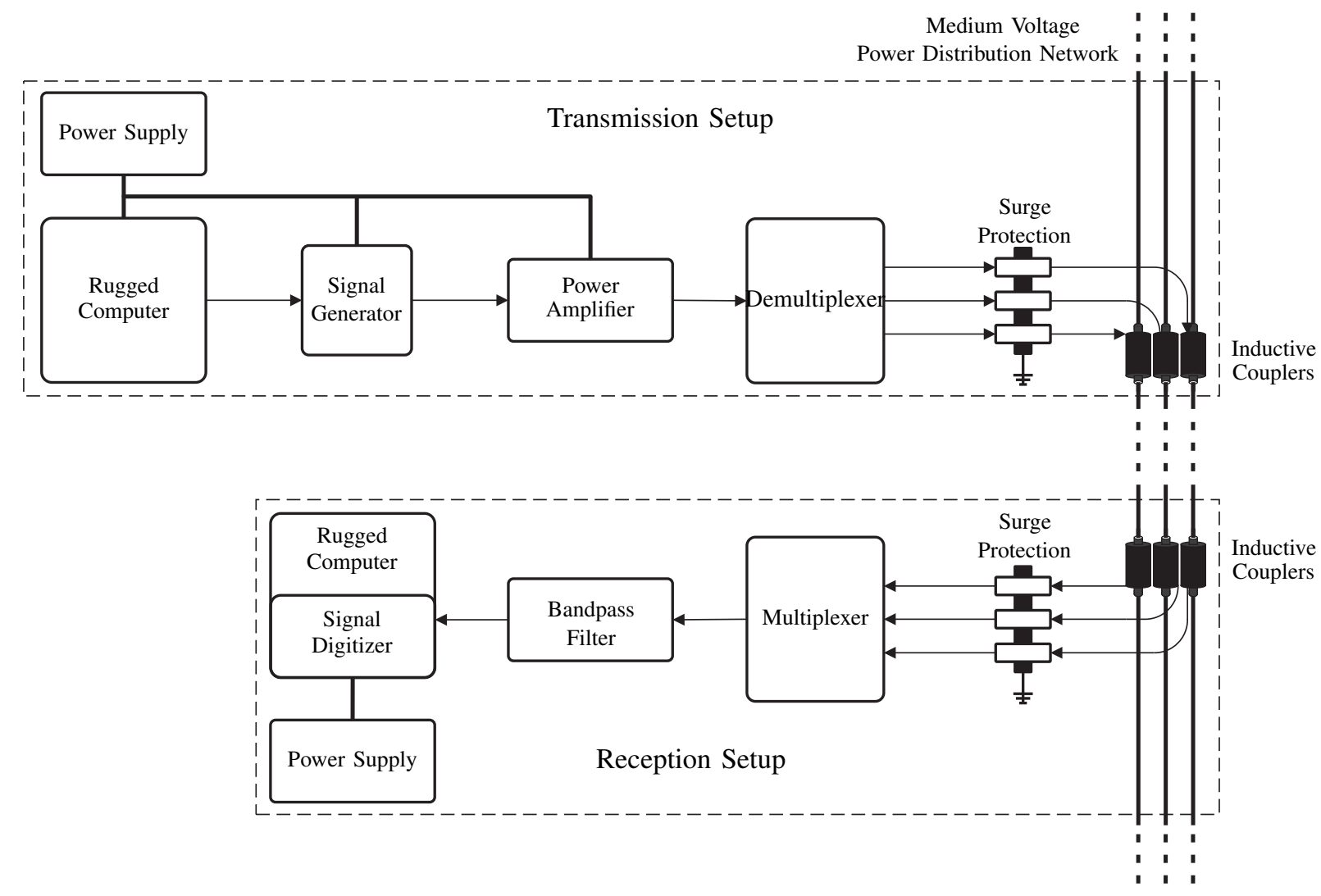

Fig. 2: The block diagrams of the transmission and reception setups.

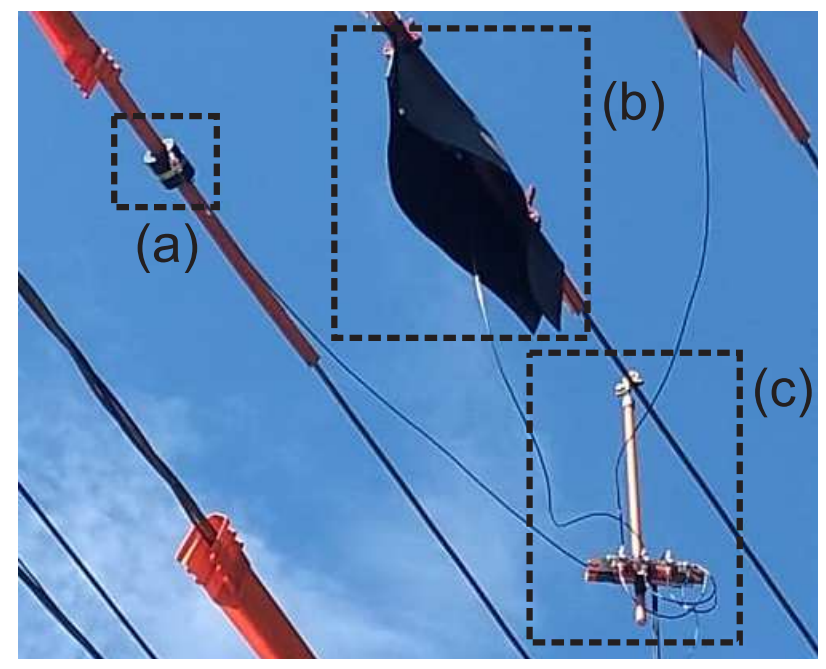

Fig. 3: The assemble of measurement instruments connected to the energized MV power cables: (a) inductive couplers, (b) a insulation blanket, and (c) the surge protection.

of power spectrum density (PSD) of the additive noise, once they allow us to quantify the usefulness of MV-PDNs as a medium for narrowband PLC systems. Also, the data rates analysis addresses phases $A, B$, and $C$ of the rural feeder.

In this section, PLC channels are modeled as time-varying and band-limited systems and their CIRs are time invariant during a time interval shorter than the coherence time. The PLC channel during the coherence time is denoted by $h(t) \in$
$\mathbb{R} \mid t \in\left[0, T_{h}\right]$, where $T_{h} \in \mathbb{R}_{+}$refers to the support of CIR of this PLC channel, and its corresponding continuous-time Fourier transform is given by $H(f) \in \mathbb{C}\left|B_{L}<\right| f \mid<B_{H}$, in which $B=B_{H}-B_{L}$ is the frequency bandwidth occupied by the PLC channel. Note that $h[n]=\left.h(t)\right|_{t=n T_{s}}$, where $n=0,1,2, \cdots, L_{h}-1$, is the discrete-time representation of $h(t)$, and owns the discrete-time Fourier transform $H\left(e^{j \omega}\right)$. In addition, $H[k], k=0,1, \cdots, 2 N-1$ is the discrete Fourier transform of the zero-padded version of the PLC channel impulse response $\{h[n]\}_{n=0}^{L_{h}-1}$ because $L_{h} \ll 2 N$, in which $N$ denotes the number of subbands of an Hermitian Symmetric Orthogonal Frequency Division Multiplexing (HS-OFDM) symbol. Also, assume that $H[k]$ is representative of $H\left(e^{j \omega}\right)$ because $N$ is large enough to ensure that each subband associated with $H[k]$ occupies a frequency bandwidth shorter than the coherence bandwidth ${ }^{2}$ of a PLC channel. Additional details are provided in [9].

\section{A. CFR Magnitude}

Fig. 4 shows the mean values of the PLC CFR average magnitudes related to the analyzed feeder. The investigated distances in the rural areas were between $2 \mathrm{~km}$ and $4.5 \mathrm{~km}$, approximately. As can be seen, the attenuation in rural can reach $55 \mathrm{~dB}$ in some frequencies, with a minimum of $-35 \mathrm{~dB}$ being observed. Also, the CFRs do not show significant spectral valleys on average. Besides, no significant differences

\footnotetext{
${ }^{2}$ The coherence bandwidth refers the maximum bandwidth in which the frequency characteristic of the channel frequency response do not change.
} 
are observed among the CFRs of different phases of the studied feeder. Thus, in general, the measured outdoor PLC channels have an approximately flat attenuation profile.

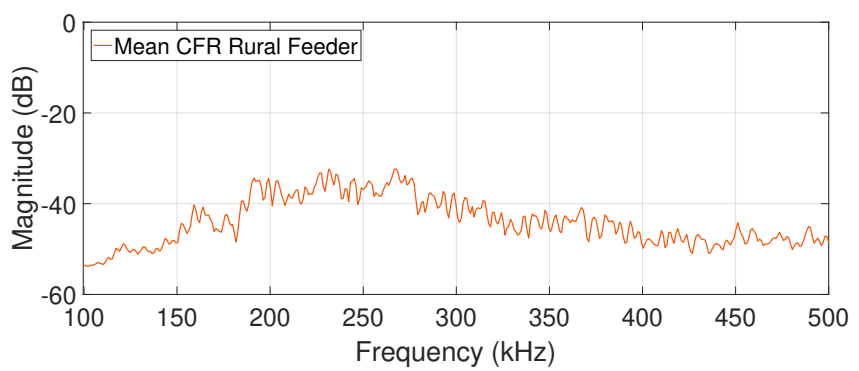

Fig. 4: The averages of magnitude of CFR of PLC channels acquired in the rural area.

Fig. 5 shows the dynamic of PLC CFR in the rural area. These plots confirm the time-invariant behavior of PLC channels obtained from MV-PDNs.

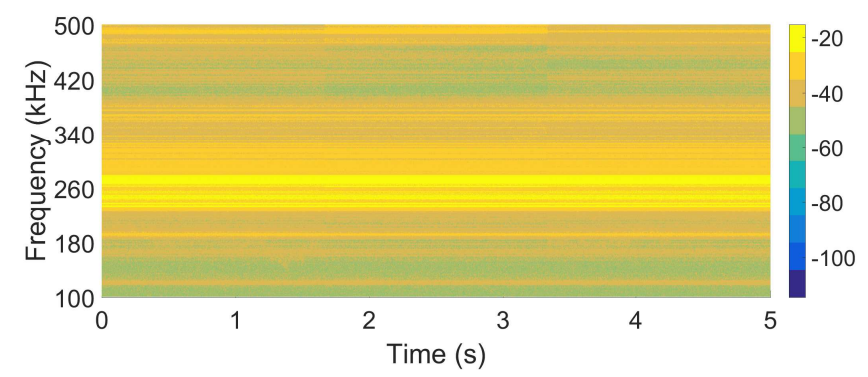

Fig. 5: Dynamic of CFR of PLC channels in MV-PDN acquired in the rural area.

\section{B. Additive Noise PSD}

The measured additive noise at different points of MV-PDNs in the rural area allowed the evaluation of PSD curves. The maximum, average, and minimum curves for the estimates of PSD are shown in Fig. 6. It can be observed that the values of PSDs range from approximately $-87 \mathrm{dBm} / \mathrm{Hz}$ up to $-77 \mathrm{dBm} / \mathrm{Hz}$ and a variation of nearly $5 \mathrm{~dB}$ can be observed among the maximum, average, and minimum curves. The results show that the PSDs can considerably vary with the choice of measurement points, mainly due to narrowband noise effects. The PSDs computed for phases $A, B$, and $C$ of the rural feeder show that insignificant differences exist among the additive noises measured in all of them.

Fig. 7 shows the spectrogram of additive noise in the rural areas. This spectogram confirm that if narrowband noise is disregarded, then the additive noise in MV-PDN can be modeled as a white Gaussian random process (i.e., $\left.\mathcal{N}\left(0, N_{0} / 2\right)\right)$. Also, $N_{0} / 2=-87 \mathrm{dBm} / \mathrm{Hz}$ can be used for generating the additive noises in rural MV-PDNs.

\section{Achievable Data Rate}

Now, assume the additive noise is a Gaussian random process and the choice of $N$ ensures the normalized signal to

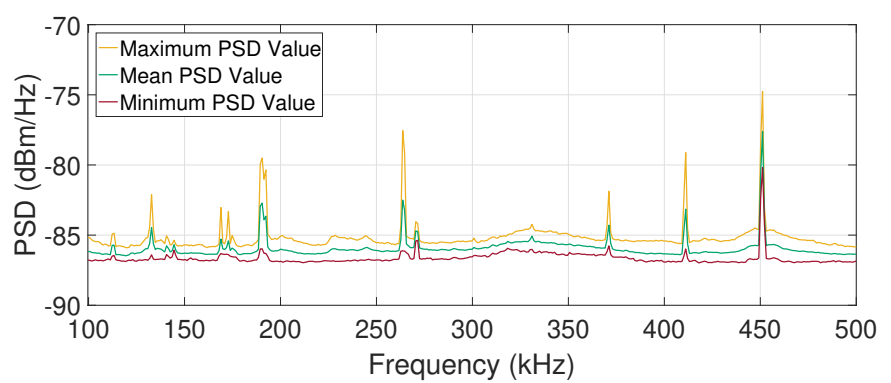

Fig. 6: PSDs of measured additive noises in MV-PDNs.

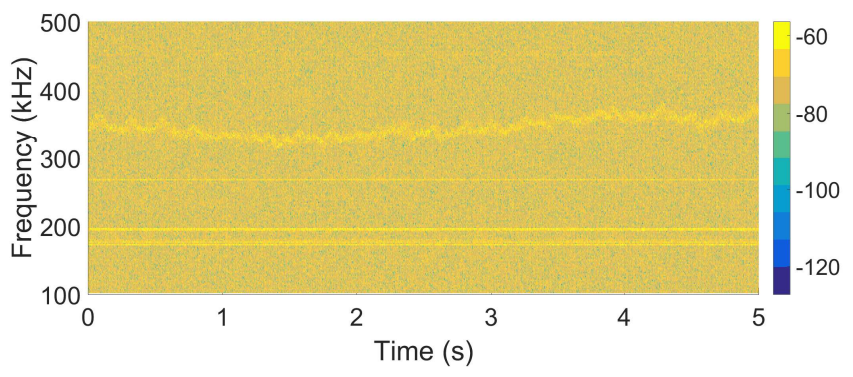

Fig. 7: Spectrogram of the additive noise in the rural area.

noise ratio be flat within the bandwidth of each subchannel. As a result, an estimate of the achievable data rate can be obtained by using

$$
R=\max _{S_{X}[k]} \frac{B}{N} \sum_{k=0}^{N-1} \log _{2}\left(1+\frac{S_{X}[k]|H[k]|^{2}}{S_{\mathcal{N}}[k]}\right)[\mathrm{bps}],
$$

subject to $2 \sum_{k=0}^{N-1}(B / N) S_{X}[k] \leq P_{X}$. Note that $S_{X}[k]$ and $S_{\mathcal{N}}[k]$ denotes the PSD of the transmitted signal in the $k^{\text {th }}$ subcarrier and the additive noise in the $k^{\text {th }}$ subchannel, respectively, while $P_{X}$ is the total transmission power. Also, the PSD of the transmitted signal varies in the interval delimited by $-60 \mathrm{dBm} / \mathrm{Hz}$ and $-20 \mathrm{dBm} / \mathrm{Hz}$, which covers regulations applied to narrowband PLC systems [7].

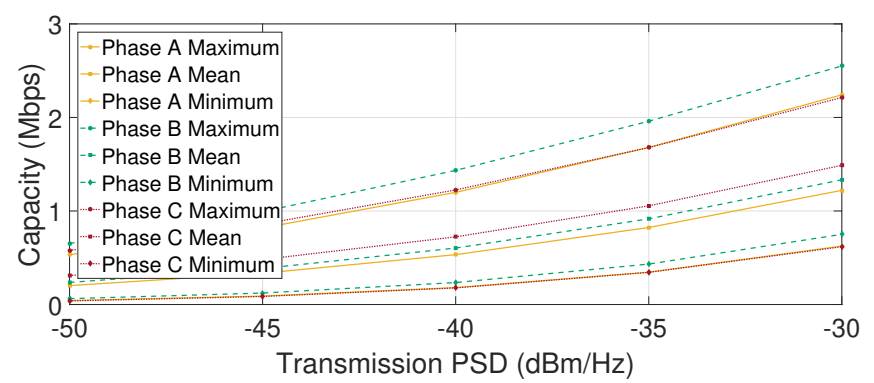

Fig. 8: Achievable data rates.

The achievable data rates related to the three phases are shown in Fig. 8. Notice that the average achievable rate in the rural feeder is around $0.9 \mathrm{Mbps}$ for a PSD of the transmitted signal equal to $-36 \mathrm{dBm} / \mathrm{Hz}^{3}$. The differences between maximum and minimum achievable data rates is

\footnotetext{
${ }^{3}$ The value $-36 \mathrm{dBm} / \mathrm{Hz}$ will result in a transmission power around $20 \mathrm{dBm}$, which is a practical amount of transmission power when the frequency bandwidth is up to $500 \mathrm{kHz}$.
} 
around 1.6 Mbps. Also, the curves inform that the achievable data rates among different phases are not relevant. In fact, the differences among the phases are lower than $0.5 \mathrm{Mbps}$ when $-36 \mathrm{dBm} / \mathrm{Hz}$ applies.

\section{Conclusions}

This paper has discussed about overhead MV-PDNs as data communication media for narrowband PLC systems based on the inductive coupling in the frequencies below $500 \mathrm{kHz}$. Relying on a measurement campaign carried out in rural areas and numerical results related to channel impulse responses and additive noises, the feasibility and adequacy of inductive coupling-based PLC systems for MV-PDNs was discussed.

Based on the raw data, it has shown that the channel impulse responses are time-invariant in overhead MV-PDNs; the additive noise can be modeled as a white Gaussian random process; and reasonable achievable data rates are observed.

\section{REFERENCES}

[1] M. Schwartz, "Carrier-wave telephony over power lines: early history of communications,” IEEE Commun. Mag., vol. 47, no. 1, pp. 14-18, Jan. 2009.

[2] T. A. Papadopoulos, C. G. Kaloudas, A. I. Chrysochos, and G. K. Papagiannis, "Application of narrowband power-line communication in medium-voltage smart distribution grids," IEEE Transactions on Power Delivery, vol. 28, no. 2, pp. 981-988, 2013.

[3] H. Hrasnica, A. Haidine, and R. Lehnert, Broadband PowerLine Communications Network Design. John Wiley \& Sons, 2004.

[4] T. Papadopoulos, B. Batalas, A. Radis, and G. Papagiannis, "Medium voltage network plc modeling and signal propagation analysis," in 2007 IEEE International Symposium on Power Line Communications and Its Applications, 2007, pp. 284-289.

[5] L. de M. B. A. Dib, V. Fernandes, M. de L. Filomeno, and M. V. Ribeiro, "Hybrid PLC/wireless communication for smart grids and Internet of Things applications," IEEE Internet Things J., vol. 5, no. 2, pp. 655-667, Apr. 2018.

[6] L. G. S. Costa, A. C. M. de Queiroz, B. Adebisi, V. L. R. da Costa, and M. V. Ribeiro, "Coupling for power line communications: A survey," Journal of Communication and Information Systems, vol. 32, no. 1, Mar. 2017.

[7] "IEEE standard for low-frequency (less than $500 \mathrm{khz}$ ) narrowband power line communications for smart grid applications," IEEE Std 1901.2-2013, pp. 1-269, 2013.

[8] G. R. Colen, L. G. Oliveira, A. J. H. Vinck, and M. V. Ribeiro, "A spectral compressive resource allocation technique for PLC systems," IEEE Trans. Commun., vol. 65, no. 2, pp. 816-826, Feb. 2017.

[9] T. R. Oliveira, C. A. G. Marques, W. A. Finamore, S. L. Netto, and M. V. Ribeiro, "A methodology for estimating frequency responses of electric power grids," J. Control Autom. Electr. Syst., vol. 25, no. 6, pp. 720-731, Dec. 2014. 